\title{
Performance Evaluation of BPSK modulation Based Spectrum Sensing over Wireless Fading Channels in Cognitive Radio
}

\author{
Ahmed Galal Ahmed Mohammed ${ }^{1}$ \\ ${ }^{I}$ (Information Technology Department, Faculty of Computers \& Information/ Cairo University, Egypt)
}

\begin{abstract}
Wireless technology is increasing rapidly over the last two decades. It has been limited to growth in wireless radio spectrum, because the radio spectrum is a finite resource which indicates that there is significant scope of improving spectrum utilization. Cognitive Radio $(C R)$ is the key enabling technology that improves the efficiency of spectrum utilization. The spectrum sensing gives the capability of CR to detect there is no primary user present in the band over wireless fading channels. In this paper we study the performance of BPSK modulation scheme under AWGN, Rayleigh, Rician and Nakagami-m fading channels are analyzed using bit error rate (BER). The performance compare between the different types of the fading channels using the $M A T L A B$ simulation. The simulation results show that the performance of the system is good in case of AWGN channel as compared to other fading channels and the Rician fading channel is better than the other fading channels such as Nakagami-m and Rayleigh fading channels when the value of $K$ is large.
\end{abstract}

Keywords: Cognitive radio, BER, BPSK, Fading channels

\section{Introduction}

Wireless technology is growing rapidly and increasing the devices that depend of the wireless technology such as cell phones, PDAs and laptops. That impact of wireless technology is much broader, e.g., through sensor networks for safety applications and home automation, smart grid control, medical wear-able and embedded wireless devices, and entertainment systems. For this explosion of wireless technology has raised a large demand for spectrum band. Cognitive radio (CR) is one critical enabling technology for future communications and networking that can utilize the limited network resources in a more efficient and flexible way without interfering with primary users. It differs from traditional communication paradigms in that the radios/devices can adapt their operating parameters, such as transmission power, frequency, modulation type, etc., to the variations of the surrounding radio environment [9].

Spectrum sensing is the basic and essential mechanisms of Cognitive Radio (CR) to find the unused spectrum band. Cognitive Radio (CR) can detect the spectrum white space, i.e., a portion of frequency band that is not being used by the primary users, and utilize the spectrum [7].

In a wireless mobile communication system, a signal can travel from transmitter to receiver over multiple reflective paths; this phenomenon is referred to as multipath propagation. The effect can cause fluctuations in the received signal's amplitude, phase, and angle of arrival, giving rise to the terminology multipath fading. There are two types of fading effects that characterize mobile communications: large-scale and small-scale fading.

$>$ Large-scale fading represents the average signal power attenuation or path loss due to motion over large areas. This phenomenon is affected by prominent terrain contours (hills, forests, billboards, clumps of buildings, etc.) between the transmitter and receiver.

$>$ Small-scale fading is also called Rayleigh fading because if the multiple reflective paths are large in number and there is no line-of-sight signal component, the envelope of the received signal is statistically described by a Rayleigh distribution. When there is a dominant non fading signal component present, such as a lineof-sight propagation path, the small-scale fading envelope is described by a Rician distribution [3].

The Nakagami-m distribution is more general and flexible distribution in the sense that it takes care of many statistical conditions by changing the value of the Nakagami parameter, $m$. When $m=1$, the Nakagami distribution becomes the Rayleigh distribution, when $m=1 / 2$, it becomes a one-sided Gaussian distribution, and when $m=\infty$ the distribution becomes an impulse (no fading) [1].

\section{Relate Works}

The authors in [2], compared the BER performance when the input of Reed-Solomon (RS) EncoderDecoder is Integer and Binary to the M-ary frequency shift keying (MFSK) under AWGN, multipath Rayleigh and Rician fading channels. The results show the binary input RS encoder has best BER performance under Rayleigh fading when the value of $\mathrm{M}$ is 2. 
In [1], the analysis performance of energy detection based spectrum sensing technique in Cognitive Radio Network over Wireless Fading channel such as AWGN, Rayleigh and Nakagami-m fading channels. The probability of detection, probability of false alarm and signal to noise ration are using to evaluate the energy detection based spectrum sensing in various ROC curves.

In [6], the performance comparison of the Rayleigh and Rician fading channels in Differential Phase Shift Key modulation. The results show the BER a very low for the same signal to noise ratio in Rician fading channels than in the Rayleigh fading channels in Differential Phase Shift Key.

In [4], the performance compare in terms of BER in OFDM using different modulation schemes such as BPSK and 16-QAM over Rayleigh, Rician and AWGN fading channels. The results are provided the QAM gives better performance under Rayleigh channel compared to other modulation schemes and channels.

In [8], the authors used the complex envelop of received signal, the level crossing rates and average fade duration on the maximal-ration combing diversity to simulate and analysis the statistical characteristics of Nakagami fading channel using MATLAB. They develop a method to simulate fading channel and comprehend the statistical performance of the Nakagami channel.

The model of the WIMAX physical layer built in [5]. They used the BER to evaluate the performance for the real data communication under AWGN, Rayleigh and Rician fading channels. They built The WIMAX communication system based on an OFDM using different channel encoding rates such as Cyclic Redundancy Check (CRC) \& Convolution Code (CC) and digital modulation schemes like QAM, 16-QAM \& 64-QAM. They demonstrate the AWGN channel has better performance than Rayleigh and Rician fading channels.

This paper is organized as follows: Section III briefly describes the system model. In Section IV we explain the probability density function (pdf) for various fading channels. Simulation results for different fading channels in Section V. Finally, in Section VI the report is summarized in the form of conclusion.

\section{System Modeling}

In Fig. 1 show the basic digital communication model. The blocks of (data source and modulator) related to the transmitter. The data source in the system model generates a stream of random bits as $0 \mathrm{~s}$ and $1 \mathrm{~s}$. The random bits are modulated as BPSK in the modulator block. After that the modulated signal passing to channel block to propagate the suitable channel which induces a Gaussian noise, Rician, Rayleigh or Nakagami$m$ fading channels in to the modulated signal. The receiver consists of demodulate block which reverses the process and extract the binary signal. It produces a sequence of random binary bits which are estimates of transmitted data.

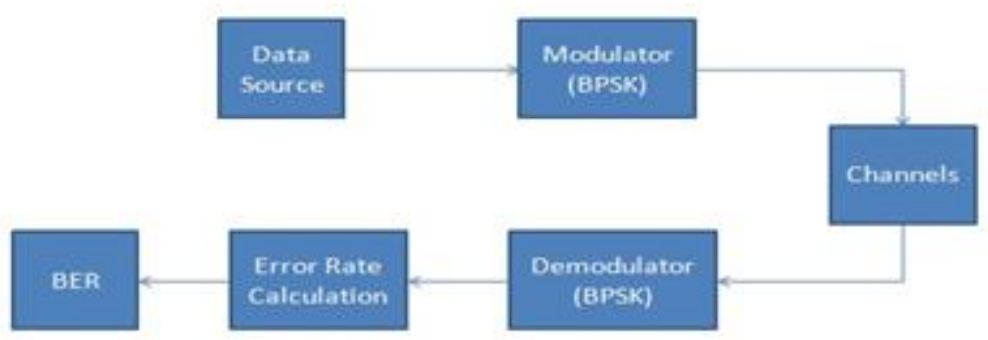

Figure 1 The system model blocks diagram

\section{A. Rayleigh fading}

\section{Channel Environment}

Rayleigh fading is a relation model when there are many objects and prominent terrain contours in the environment that reflect, diffract and scatter that propagate multipath of the transmitted signal before it receives at the receiver [3][4].

When there are large numbers of reflective paths and there is no line-of-sight communication between the transmitter and the receiver, the envelope of the received signal is statistically describing a Rayleigh PDF, expressed as:

$$
p(r)= \begin{cases}\frac{r}{\sigma^{2}} \exp \left[-\frac{r^{2}}{2 \sigma^{2}}\right] & \text { for } r \geq 0 \\ 0 & \text { otherwise }\end{cases}
$$

where $\mathrm{r}$ is envelope amplitude of the received signal, and the $2 \sigma^{2}$ is the pre-detection mean power of the multipath signal. 


\section{B. Rician Fading}

The Rician fading is different from Rayleigh fading the Rician assumes a direct line-of-sight path between the transmitter and the receiver much stronger than the multipath signals that arrive to the receiver. The Rician PDF can be written as [5]:

$$
p(r)=\frac{r}{\sigma^{2}} \exp \left(-\frac{r^{2}+A^{2}}{2 \sigma^{2}}\right) I_{0}\left(\frac{r A}{\sigma^{2}}\right), r \geq 0, A \geq 0
$$

where $\sigma^{2}$ is the variance in-phase and quadrature components. A is the amplitude of the signal of the dominant path and $\mathrm{I}_{0}$ is zero-order modified Bessel function of the first kind.

\section{Nakami- $m$ Fading}

Nakagami distribution doesn't assume line-of-sight conditions, but use a parametric gamma distribution based density function to describe the experimental data and get approximately distribution, so it is more universality, the PDF of Nakagami distribution is [8]:

$$
p(r)=\frac{2 m^{m} r^{2 m-1}}{\Omega^{m} \Gamma(m)} \exp \left(-\frac{m^{2}}{\Omega}\right) \quad m \geq \frac{1}{2}, \quad r \geq 0
$$

where $\mathrm{m}$ is Nakagami parameter, described the fading degree of propagation field due to scattering and multipath interference process, and $\Omega$ is the average power of multipath scatter field, $\Gamma(\mathrm{m})$ is the gamma function. The relationship between the Rician fading factor $k$ and the Nakagami- $m$ fading factor $m$ is [1]:

\section{AWGN Channel}

$$
K=\frac{\sqrt{m^{2}-m}}{m-\sqrt{m^{2}-m}}, \quad m=\frac{(K+1)}{(2 K+1)}
$$

AWGN is a noise that affects the transmitted signal when it passes through the channel. AWGN channel is not associated with either fading or any other system parameters. It is just the noise that is added to the signal when it is traveling through the channel [4][5]. The AWGN channel is represented by:

$$
r(t)=s(t)+n(t)
$$

where $s(t)$ is transmitted signal and $n(t)$ is white Gaussian noise. The values of white Gaussian noise at any pair of times are identically distributed and statistically independent on each other.

\section{Simulation Results}

This section we present and evaluate the results using the Matlab R2012a. The results are used the ratio of signal energy per bit to noise power density $E_{b} / N_{0}$ and bit error rate BER to evaluate and analyze the performance of BPSK over AWGN, Rayleigh, Rician, and Nakagami- $m$ fading channels.

In the Fig.2, Fig.3 and Fig.4 show the BER performance and the effect the AWGN, Rayleigh, Rician with $(K=1,2,4,8)$ and Nakagami- $m$ with $(m=1.333,1.8,2.778,4.764)$ over BPSK. In the all figures we can see that the AWGN channel has lower BER than different fading channels this can see very clear in the Fig.5.

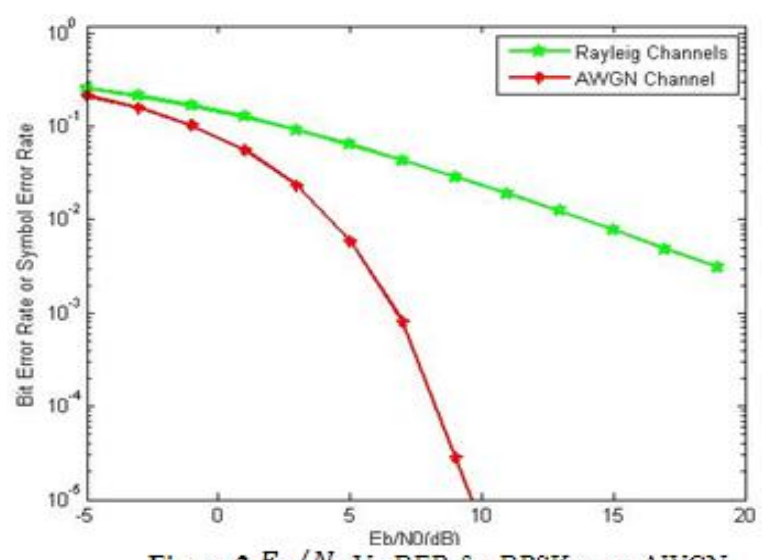

Figure $2 E_{b} / N_{0}$ Vs BER for BPSK over AWGN and Rayleigh Channel.

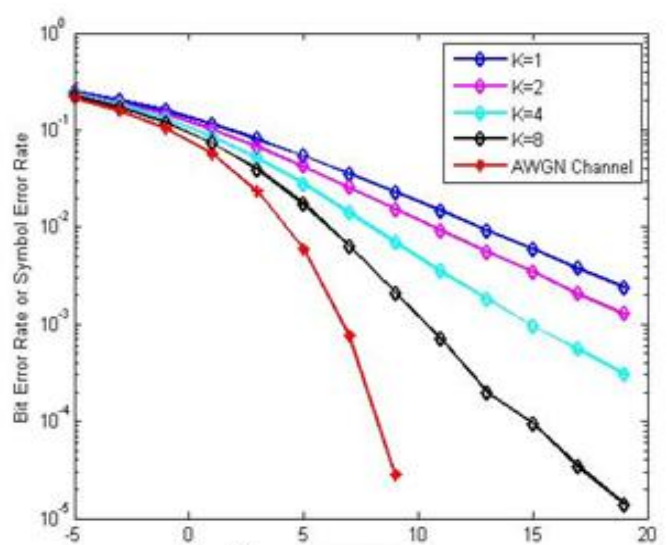

Figure $3 E_{b} / N_{0} V_{s}$ BER for BPSK over AWGN and Rician Channels $(\mathrm{K}=1,2,4,8)$ 


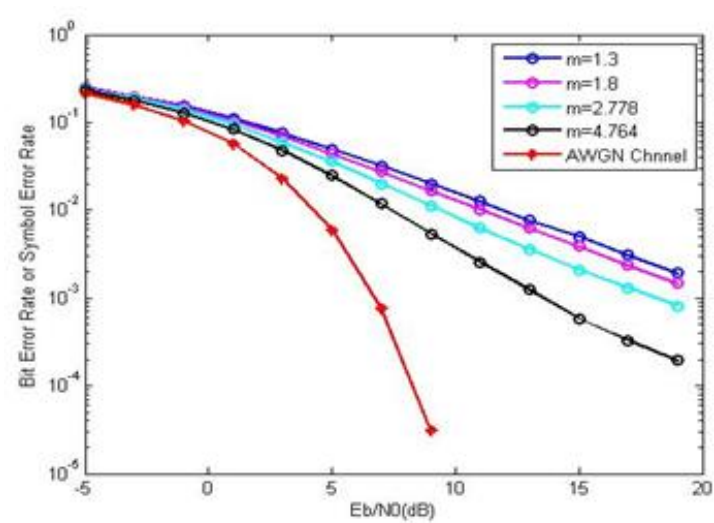

Figure $4 E_{b} / N_{0}$ Vs BER for BPSK over AWGN and Nakagami-m Channels $(\mathrm{m}=1.333,1.8,2.778,4.764)$

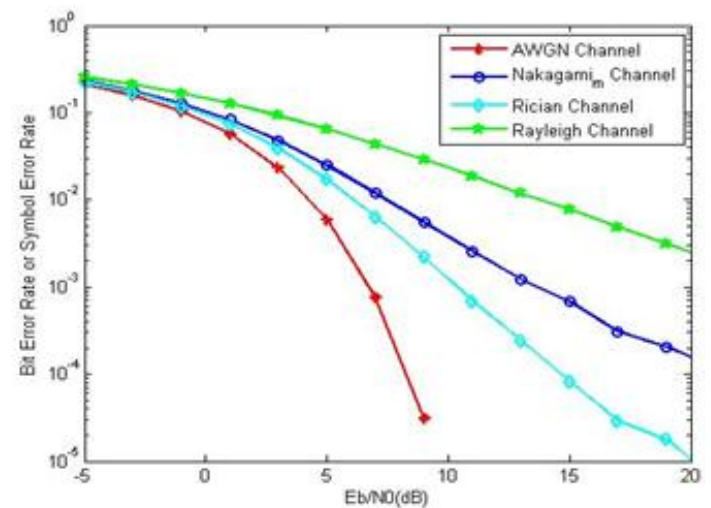

Figure $5 E_{b} / N_{0}$ Vs BER for BPSK over AWGN, Rician Channels $(\mathrm{K}=8)$, Nakagami-m Channels $(\mathrm{m}=4.764)$, and Rayleigh Channel

We can demonstrate again from the Fig.5 the Rayleigh fading channel has higher BER than Rician and Nakagami-m fading channels. The Rician and Nakagami-m fading channels we find them between the AWGN and Rayleigh channel. They depend to the value of $K$ 's and $m$ 's we can see that in the following figures.

The figures from Fig.6 to Fig.9 show the BER performance of the Rician channel decreases when increasing the value of $K$ factor from 1 to 8 in BPSK and give us better result than Nakagami- $m$ this match the results in the Fig. 10 when using Monte Carlo simulation in the Matlab for BPSK under Rician channel when the values of $K$ are 1, 2, 4 and 8 for the range of $E_{b} / N_{0}$ from -5 to 20. By using the relation between Nakagami$m$ and Rician channel in Eq.4 the values of $m$ in the Nakagami- $m$ channel to be 1.333, 1.8, 2.778 and 4.764.

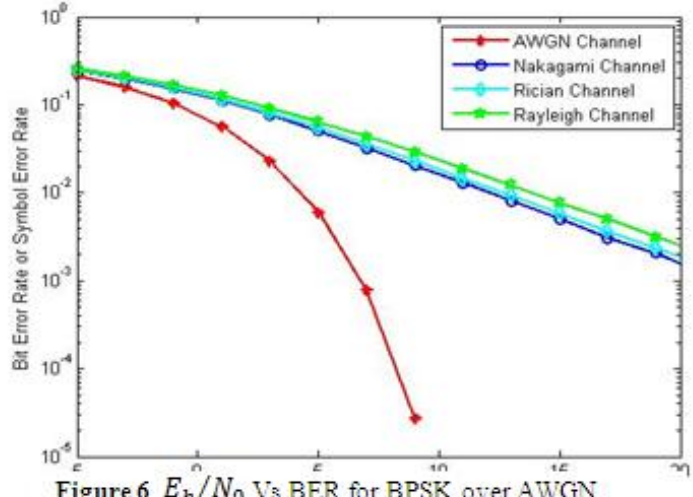

Figure $6 E_{b}^{n} / N_{0}$ Vs BER for BPSK over AWGN,

Rician Channels $(\mathrm{K}=1)$, Nakagami-m Channels ( $\mathrm{m}=1.3)$, and Rayleigh Channel

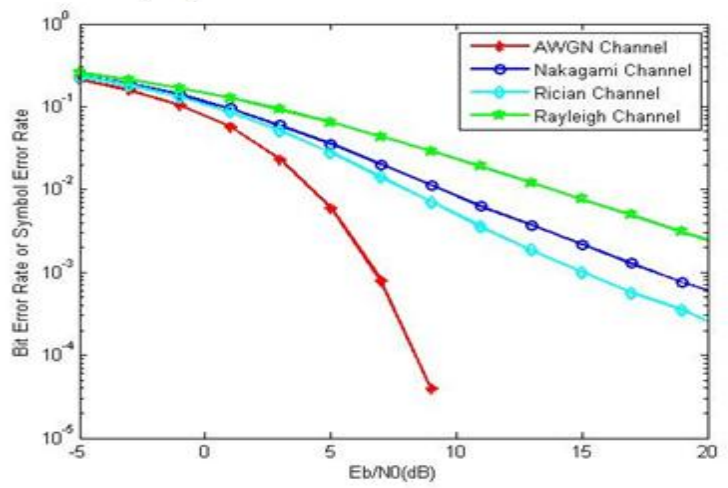

Figure $8 E_{b} / N_{0} V_{s}$ BER for BPSK over AWGN

Rician Channels $(K=4)$, Nakagami-m Channels $(m=2.778)$, and Rayleigh Channel

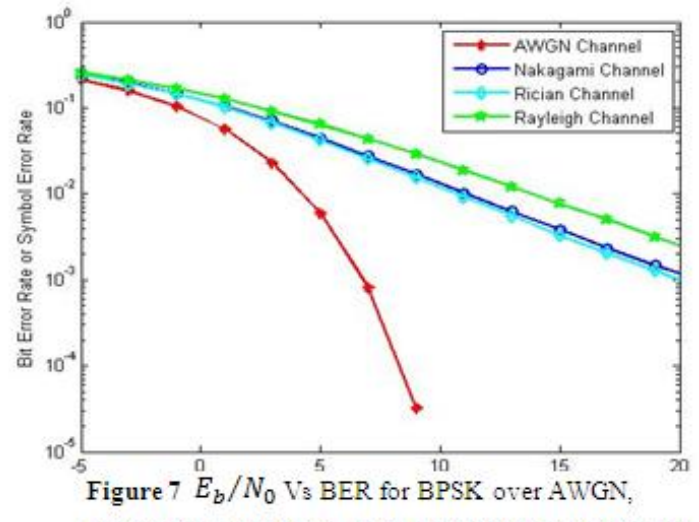

Rician Channels ( $\mathrm{K}=2)$, Nakagami-m Channels ( $m=1.8)$, and Rayleigh Channel

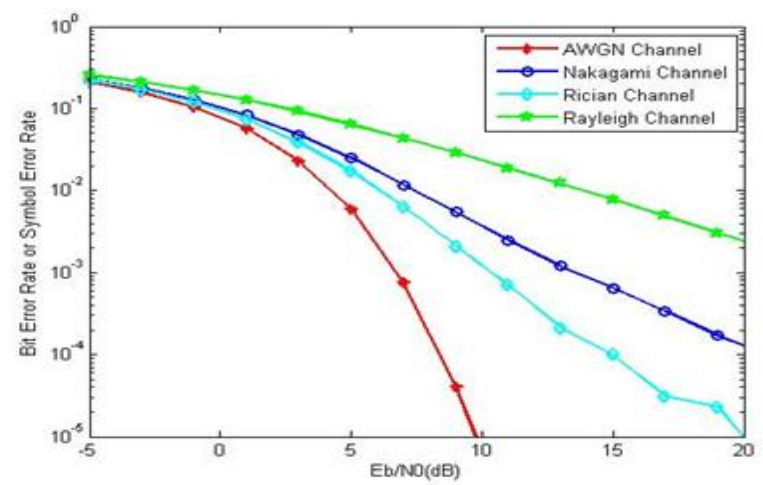

Figure $9 E_{b} / N_{0} V_{s}$ BER for BPSK over AWGN

Rician Channels ( $K=8)$, Nakagami-m Channels $(m=4.764)$, and Rayleigh Channel 


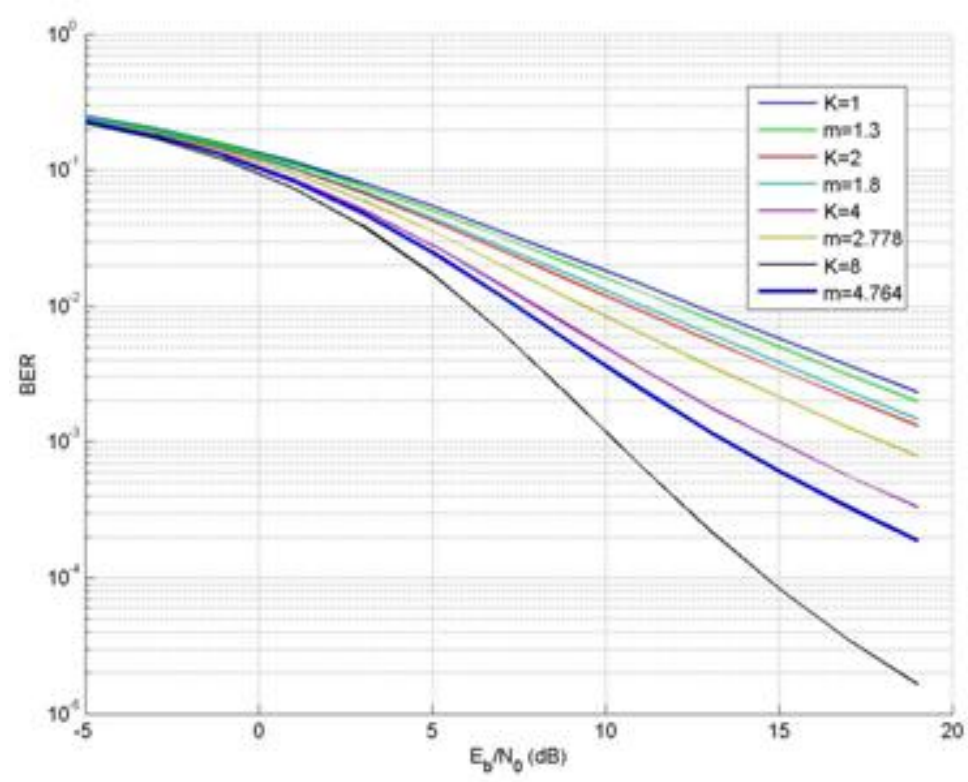

Figure 10 Comparison between Nakagami- $m$ and Rician Channel in Monte Carlo simulation

\section{Conclusion}

In this paper we compare the performance in terms of BER using BPSK modulation on different fading channels. The simulation results are provided and from which we can evidently conclude that the BPSK gives better performance under AWGN channel compared to other fading channels and the Rayleigh is the worst performance case. Finally, we analyze the performance of BPSK modulation under Nakagami- $m$ and Rician channels and show that the system exhibits the improve performance in the Rician channel when increasing the $K$ factor value as compare to value of $\mathrm{m}$ factor in Nakagami- $m$ channel.

\section{References}

[1]. V. M. K. a. O. S. Vaidya, "Analysis of Energy Detection based Spectrum Sensing over Wireless Fading Channels in Cognitive Radio Network," International Journal of Emerging Technology and Advanced Engineering, vol. 2, no. 3, pp. 645-653, 2013.

[2]. T. S. a. D. A. Nachiappan, "An Insight into BER Performance of Reed-Solomon coded M-FSK under AWGN, Rayleigh and Rician Fading Channels," International Journal of Advanced Research in Electrical, Electronics and Instrumentation Engineering, vol. 2, no. 9, pp. 1488-1492, 2013.

[3]. S. B., "Rayleigh fading channels in mobile digital communication systems .I. Characterization," Communications Magazine, IEEE, vol. 35 , no. 7 , pp. $90-100,1997$.

[4]. S. K. B. a. S. K. Chaparala, "Performance Evaluation of OFDM System with Rayleigh,Rician and AWGN channels," International Journal of Emerging Technology and Advanced Engineering, vol. 3, no. 3, pp. 678-682, 2013

[5]. M. M. R. M. A. I. a. A. T. I. Nuzhat Tasneem Awon, "Effect of AWGN \& Fading (Raleigh \& Rician)channels on BER performance of a WiMAX communication System," International Journal of Computer Science and Information Security (IJCSIS), vol. 10, no. 8, pp. 11-17, 2012.

[6]. D. a. M. \{Mr.P.Sunil Kumar, "Performance evaluation of Rayleigh and Rician Fading Channels using M-DPSK Modulation Scheme in Simulink Environment," International Journal of Engineering Research and Applications(IJERA), vol. 3, no. 3, pp. 13241330,2013

[7]. M. A. A. a. Z. A. Hussein, "ENERGY DETECTION TECHNIQUE FOR SPECTRUM SENSING IN COGNITIVE RADIO: A SURVEY," International Journal of Computer Networks and Communications (IJCNC), vol. 4, no. 5, pp. 1 - $20,2012$.

[8]. L. T. a. Z. Hongbo, "Analysis and simulation of Nakagami fading channel with MATLAB," in Environmental Electromagnetics, 2003. CEEM 2003. Proceedings. Asia-Pacific Conference on, Hangzhou, China, 2003.

[9]. K. Beibei Wang and Liu, "Advances in cognitive radio networks: A survey," Selected Topics in Signal Processing, IEEE Journal, vol. 5 , no. 1 , pp. $5-23,2011$. 